\title{
Quantum-behaved Particle Swarm Optimization for Power Economic Dispatch Problem of Units with Multiple Fuel Options
}

\author{
Chao-Lung Chiang
}

\begin{abstract}
This paper presents a quantum-behaved particle swarm optimization (QPSO) with a multiple updating (MU) for solving the power economic dispatch problem (PEDP) of generators with multiple fuel options (MFOs). The QPSO assists the proposed method efficaciously find and precisely search. The MU helps the proposed method prevent deforming the augmented Lagrange function (ALF) and caused difficultly in searching optimal solution. The proposed approach combines the QPSO and the MU that has benefits of adopting a widespread area of punishment parameters and a small-size population. The proposed algorithm has been demonstrated on a practical ten generating units system; every one unit is composed of two or three fuel changes. The entire fuel price got by the proposed QPSO-MU has been competed with former studies for validating its efficacy. Compared achievements clearly express that the presented method is an effective alternative for resolving PEDP of units with MFOs in the realistic operations of power system.
\end{abstract}

Index Terms-Augmented Lagrange Function; Economic Dispatch; MFOs, Particle Swarm Optimization.

\section{INTRODUCTION}

Generally, power economic dispatch problem (PEDP) decides the best electricity productivities for all power generators, which will reduce the entire charge while the system both of demand and constraints have been contented [1], [2]. PEDP is an integration problem due to its huge size, a nonlinear objective function, and a big amount of system constraints. Traditional methods of lambda iterative skill and quadratic programming provide better outcomes, but when the searching area is nonlinear characteristic and has convex feature they become more complex with a sluggish convergency and don't guarantee to find the best answer.

In practical operations of power system, some fired generators, which are provided with diverse fuels like natural gas, oil and coal. Functions of fuel price are always discontinuous and segmented as piecewise quadratic cost functions for MFOs. The PEDP of generators having MFOs is to reduce entire fired cost between available fired sources for each generator meeting loading and generation ranges. The PEDP is a nonconvex and complex optimal issue because it involves segmented values at each limit constituting numerous local optima. Consequently, traditional approaches are usually hard to handle the PEDP. A traditional method [3] linearized segments of cost function to resolve the PEDP of generators having MFOs. A numerical method (HM) [4] retained the supposition of piecewise quadratic price curves and resolve the PEDP with MFOs. Nevertheless, the computation of HM was still exponentially growing time complexities for bigger systems with nonconvex constraints. The Hopfield neural network (HNN) [5] was also ingenious to punishment factors correlative with system constraints. The enhanced Lagrangian neural network (ELANN) [6] had speedy computational efficacy because a momentum skill was used in learning procedure of ELANN. Nevertheless, approaches of HNN and ELANN needed a big iterative loops for convergency of the best answer, and they usually expressed oscillation in the transient procedure. The Adaptive Hopfield neural network (AHNN) [7], [8] always adjusts the gradient and biases of neurons within the convergent procedure to promote the computational property.

Some deductive optimal skills have been utilized to resolve the PEDP with MFOs system, too. The genetic algorithm (GA) [9], [10], evolutionary programming (EP) [11], [12], differential evolution (DE) [13], particle swarm optimization (PSO) [14], a combination of the EP, tabu search and quadratic programming (ETQ) [15], the hybrid real coded genetic algorithm (HRCGA) [16], and the hybrid integer coded differential evolution-dynamic programming (HICDEDP) [17]. Nevertheless, methods mentioned above are usually tardy because of combining single techniques.

In the near future, various algorithms also have been presented to solve PEDP of units with MFOs. Such as a synergic predator-prey optimization (SPPO) [18], an integrated of modified shuffled frog leaping algorithm (MSFLA) and global-best harmony search algorithm (GHS), MSFLA-GHS [19], augmented Lagrange Hopfield network initialized using quadratic programming (QP-ALHN) [20], improved particle swarm optimization (IPSO) [21], enhanced augmented Lagrange Hopfield network (EALHN) [22], composite cost function (CCF) [23], and chaotic improved honey bee mating optimization (CIHBMO) [24].

PSO has been diffusely used in power optimal problems. Even if this method can bring about high-quality answers with less calculation iterations and steady convergency contrast to other algorithms [25], it appears to be ingenious to tuning of certain weightings or factors. PSO exploited from Kennedy et al. [26], its developing was based on observations of animals' societal actions, such as bird flocking, fish schooling, and swarm concept. PSO has shown its simplicity 
and facile realization in many previous reports [27]-[35]. Nevertheless, the global convergent characteristic of PSO cannot be ensure to obtain. For conquering the drawback of PSO, quantum-behaved particle swarm optimization (QPSO) is presented [36]-[39], and this method has attracted more and more attention lately [40]-[44]. The QPSO has been confirmed the truth of its global convergence in accordance with the explanation from [37].

\section{SYSTEM FORMULATION}

The objective function of PEDP is mainly to decide the best loading for all online dispatching generators, which reduces the entire fired price while approving of system constraints. It can be described below.

\section{A. Fuel Cost}

Generally, fuel cost function of every power generating unit is denoted by a sole quadratic cost function. Herein, because of MFOs, this price function is piecewise and quadratic. Accordingly, the PEDP of generators having MFOs with piecewise quadratic price functions are expressed as [5]

$$
\begin{aligned}
& \min f=\sum_{j=1}^{n} F_{j}\left(P_{j}\right) \\
& F_{j}\left(P_{j}\right)=\left\{\begin{array}{llll}
a_{j 1}+b_{j 1} P_{j}+c_{j 1} P_{j}^{2}, & \text { fuel } 1, & P_{j} \leq P_{j} \leq P_{j 1} \\
a_{j 2}+b_{j 2} P_{j}+c_{j 2} P_{j}^{2}, & \text { fuel } 2, & P_{j 1} \leq P_{j} \leq P_{j 2} \\
\vdots & & & \\
a_{j k}+b_{j k} P_{j}+c_{j k} P_{j}^{2}, & \text { fuel } & k, & P_{j k-1} \leq P_{j} \leq \bar{P}_{j}
\end{array}\right.
\end{aligned}
$$

Where $F_{j}\left(P_{j}\right)$ represents fuel price of generating unit $j . P_{j}$ expresses power output of generating unit $j . a_{j k}, b_{j k}$ and $c_{j k}$ indicate cost coefficients of unit $j$ for using fuel kind $k . n$ is entire amount of generating units.

\section{B. System Balance Constraint}

The entire generation must be match to the summation of system demand and transmission loss [1]

$$
\sum_{j=1}^{n} P_{j}-P_{D}-P_{L}=0
$$

Where $P_{D}$ is the entire system demand and $P_{L}$ is the transmission loss.

\section{Capacity Limit Constraints}

The power productivity of every generating unit $j$ should be between its minimum $\underline{P}_{j}$ with maximum $\bar{P}_{j}$, and it can be displayed as [1]

$$
\underline{P}_{j} \leq P_{j} \leq \bar{P}_{j}
$$

\section{THE PROPOSED AlgORITHM}

\section{A. $\quad$ PSO}

The PSO [25], [26] is an iterative algorithm based on the searching action of a swarm particles in a multidimensional search area. In PSO, the velocity and the position of each particle are updated. In the light of the fitness of the updated individuals, the personal best position of each particle and the global best position in all the particles are updated. Regarding the update of the velocities in the PSO, a particle is affect by its personal best position and the global best position. Thus, the PSO finds the global optimum by regulating the trajectory of each particle toward its personal best position and the global best position. Nevertheless, it still remain drawbacks of converging to local optimal answers. In PSO with $m$ individuals, each individual is treated as a volume-less particle in the $n$-dimensional space, with the position vector and velocity vector of particle $i$ at the $t$ th generation expressed as $x_{i}(\mathrm{t})=\left[x_{i, 1}(t), x_{i, 2}(t), \ldots, x_{i, n}(t)\right]$ and $v_{i}(t)=\left[v_{i, 1}(t), v_{i, 2}(t), \ldots\right.$, $\left.v_{i, n}(t)\right]$. The particle shifts in accordance with the following equations [25]:

$$
\begin{gathered}
v_{i, j}(t+1)=w \cdot v_{i, j}(t)+c_{1} \cdot r_{1, j}(t) \cdot\left\lfloor\operatorname{pbest}_{i, j}(t)-x_{i, j}(t)\right\rfloor \\
+c_{2} \cdot r_{2, j}(t) \cdot\left[\operatorname{gbest}_{j}(t)-x_{i, j}(t)\right] \\
x_{i, j(t+1)}=x_{i, j}(t)+v_{i, j}(t+1)
\end{gathered}
$$

for $i=1,2, \ldots, m ; j=1,2, \ldots, n$, where $c_{1}$ and $c_{2}$ are called acceleration coefficients, and $w$ is the inertia weight. Vector $\operatorname{pbeset}_{i, j}(t)=\left[\operatorname{pbest}_{i, 1}(t), \operatorname{pbest}_{i, 2}(t), \ldots, \operatorname{pbest}_{i, n}(t)\right]$ is the best former position of particle $i$ called personal best (pbest) position, and vector gbest $(t)=\left[\operatorname{gbest}_{1}(t), \operatorname{gbest}_{2}(t), \ldots\right.$, $\left.\operatorname{gbest}_{n}(t)\right]$ is the position of the best particle between all the particles in the population and called global best (gbest) position.

\section{B. $Q P S O$}

A major drawback of PSO is not guaranteed to be global convergency [25], and PSO is susceptible to trap into local optima even if it converges quickly. Trajectory analysis illustrates the fact that convergence of the PSO algorithm may be realized if every particle converges to its local attractor $P_{i, j}(t)$, defined at the coordinates [45]:

$$
P_{i, j}(t)=\phi_{j}(t) \cdot \operatorname{Pbest}_{i, j}(t)+\left[1-\phi_{j}(t)\right] \cdot \operatorname{gbest}_{j}(t)
$$

Where $\phi_{j}(t)=c_{1} * r_{1, j}(t) /\left[c_{1}(t) * r_{1, j}(t)+c_{2} * r_{2, j}(t)\right]$ concerning the random numbers $r_{l, j}(t)$ and $r_{2, j}(t)$ in Eq. (5). Because the acceleration coefficients $c_{1}$ and $c_{2}$ in the PSO are usually formulated to be equal, i.e. $c_{1}=c_{2}, \phi_{j}(t)$ will be a sequence of uniformly distributed random number within $(0,1)$, that is $\phi_{j}(t) / U(0,1)$. In the QPSO, it is assumed that every single particle is spin-less one with quantum behavior. Therefore, state of the particle is characterized by wave function $w$, where $|w|^{2}$ is the probability density function of its position. We suppose that, at the $t$ th generation, particle $i$ moves in $n$ dimensional area with a $d$ potential well at $P_{i, j}(t)$ on the $j$ th dimension $(1 \leq j \leq n)$ to ensure the convergency. Using the Monte Carlo method, the particle shifts in the light of later iterative equations [36]-[38]:

$\left\{\begin{array}{lll}x_{i, j}(t+1)=P_{i, j}(t)+\alpha \cdot\left|C_{j}(t)-x_{i, j}(t)\right| \cdot \ln \left(1 / r_{3}\right), & \text { if } & r_{4} \geq 0.5 \\ x_{i, j}(t+1)=P_{i, j}(t)-\alpha \cdot\left|C_{j}(t)-x_{i, j}(t)\right| \cdot \ln \left(1 / r_{3}\right), & \text { if } & r_{4}<0.5\end{array}\right.$

Where $r_{3}$ and $r_{4}$ are values generated in accordance with a uniform probability distribution within range $(0,1)$, and $C$ is called mean best position defined as the average value of the 
pbest positions of all particles:

$$
C_{j}(t)=(1 / m) \sum_{i=1}^{m} \operatorname{pbest}_{i, j}(t)
$$

A factor $\alpha$ in Eq. (8) is called contraction-expansion (CE) coefficient, which can be tuned to control the convergent rate of the particle. Previous achievements have demonstrated that the factor $\alpha$ have to be set as $\alpha<1.782$, thus, convergency or boundedness of the particle could be ensured [38]. Generally, it can guide the QPSO to a favorable property on the whole to change the value of a linearly between $\alpha_{0}$ and $\alpha_{1}\left(\alpha_{0}<\right.$ $\alpha_{1}$ ) in carrying out the QPSO procedure.

\section{C. $M U$}

Michalewicz and Schoenauer [46] investigateed and emulated many constraint-handling methods employed in evolutionary algorithms. Within these methods, the punishment function technique is one of the highly prevalently applied to mange system constraints, and the objective function involves a punishment function that is constituted of the squared or absolute constraint violation terms. Powell [47] indenoted that traditional optimal approaches comprise a punishment function have some disadvantages that become extremely severe when punishments are big. Moreove, big punishments illconditioned the punishment function so that finding a better answer is hard. Nevertheless, if the punishments are too small, the constraint violation does not dedicate a big cost to the punishment function. Hence, how to choose suitable punishments is not inessential. Thus, the MU [48] is presented to manage this constrained optimization problem. This algorithm can surmount the ill-conditioned characteristic of the objective function. Thinking about a nonlinear optimiztion system with general constraints as follows:

$$
\begin{array}{lll} 
& \min _{x} f(x) \\
\text { subject to } \quad & h_{k}(x)=0, \quad k=1, \ldots, m_{e} \\
& g_{k}(x) \leq 0, \quad k=1, \ldots, m_{i}
\end{array}
$$

Where $x$ stands a $n_{C}$-dimensional variables, and $h_{k}(x)$ and $g_{k}(x)$ respectively express constraints of equality and inequality. The punishment terms as well as Lagrange function are integrated in the augmented Lagrange function (ALF) [47] as follows:

$$
\begin{aligned}
L_{a}(x, v, v)= & f(x)+\sum_{k=1}^{m_{e}} \alpha_{k}\left\{\left[h_{k}(x)+v_{k}\right]^{2}-v_{k}^{2}\right\} \\
& +\sum_{k=1}^{m_{i}} \beta_{k}\left\{\left\langle g_{k}(x)+v_{k}\right\rangle_{+}^{2}-v_{k}^{2}\right\}
\end{aligned}
$$

Where $\alpha_{k}$ and $\beta_{k}$ are the positive punishments, and the relevant Lagrange multipliers $v=\left(v_{1}, \ldots, v_{m_{e}}\right)$ and $v=\left(v_{1}, \ldots, v_{m_{i}}\right) \geq 0$ are respectively related with constraints of equality and inequality. The adumbration of the ALF doesn't modify appearance among generations while system constraints of PEDP are linear characteristics. Consequently, the figure of the ALF is just biased or shifted in relation to the original objective function, $f(x)$. Therefore, small punishment can be used in the MU. Nevertheless, the appearance of adumbration of $L_{a}$ is modified by punishments while the system constraints of PEDP have nonlinear characteristics, illustrating that big punishments also bring about certain difficulties in computation process. Adaptive punishments of the MU are used to moderate difficulties mentioned above. Many detailed information of the MU have demonstrated in previous research of [48].

\section{The proposed QPSO-MU}

Fig. 1 has shown the flow diagram of the proposed QPSOMU, which has two iterative of outer and inner loops. The ALF is employed to find a smallest value in the inner loop with the given punishments and multipliers, which are then updated in the outer loop to yielding an upper limit of $L_{a}$. When both inner and outer iterative loops turned into enoughly big, the ALF function will be converging on a saddle-point of the dual problem [46]. The suggested QPSOMU has merits of an efficient search the optimal answer of economic dispatch in the inner loop and the MU validly manages constraints of EEDP system in outer loop.

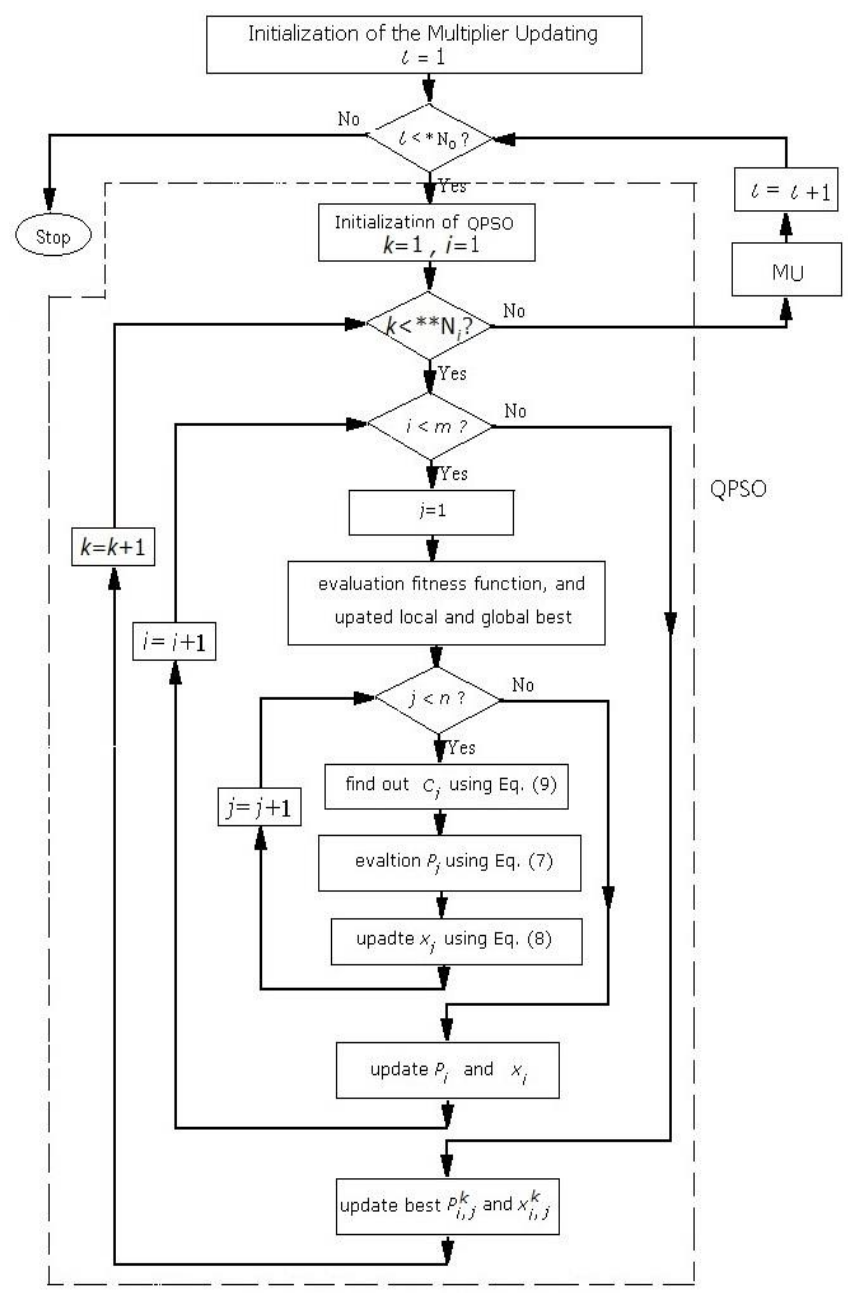

Fig. 1. The Flow Chart of the QPSO-MU

${ }^{*} \mathrm{~N}_{\mathrm{o}}$ : Maximum number of iterations of outer loop

${ }^{* *} \mathrm{~N}_{\mathrm{i}}$ : Maximum number of iterations of oinner loop 
TABLE1: COMPARES FIFTEEN COMPUTATIONAL RESULTS OBTAINED FROM PREVIOUS METHODS AND THE PROPOSED QPSO-MU.

\begin{tabular}{|c|c|c|c|c|c|c|c|c|c|c|c|}
\hline $\begin{array}{l}\text { Generation } \\
(\mathrm{MW})\end{array}$ & $F T$ & $\begin{array}{c}\text { SPPO } \\
{[18]}\end{array}$ & $\begin{array}{c}\text { SFLA- } \\
\text { GHS } \\
{[19]}\end{array}$ & $\begin{array}{c}\text { QP- } \\
\text { ALHN } \\
{[20]}\end{array}$ & $\begin{array}{c}\text { IPSO } \\
{[21]}\end{array}$ & $\begin{array}{c}\text { EALHN } \\
{[22]}\end{array}$ & $\begin{array}{l}\mathrm{CCF} \\
{[23]}\end{array}$ & $\begin{array}{c}\text { CIHMBO } \\
{[24]}\end{array}$ & $\begin{array}{c}\text { AHNN } \\
\text { [7] }\end{array}$ & $\begin{array}{c}\mathrm{HM} \\
{[4]}\end{array}$ & $\begin{array}{c}\text { QPSO- } \\
\text { MU }\end{array}$ \\
\hline $\mathrm{P}(\mathrm{G} 1)$ & 2 & 218.3507 & 218.2499 & 218.250 & 218.235 & 218.2502 & 218.2499 & 218.1050 & 225.7 & 218.4 & 218.7717 \\
\hline $\mathrm{P}(\mathrm{G} 2)$ & 1 & 211.5895 & 211.6626 & 211.663 & 211.706 & 211.6627 & 211.6626 & 211.6596 & 215.2 & 211.8 & 212.2125 \\
\hline $\mathrm{P}(\mathrm{G} 3)$ & 1 & 280.7704 & 280.7228 & 280.723 & 280.721 & 280.7230 & 280.7228 & 280.6571 & 291.8 & 281.0 & 280.8796 \\
\hline $\mathrm{P}(\mathrm{G} 4)$ & 3 & 239.6790 & 239.6315 & 239.632 & 239.609 & 239.6316 & 239.6315 & 239.6864 & 242.3 & 239.7 & 239.4832 \\
\hline $\mathrm{P}(\mathrm{G} 5)$ & 1 & 278.4467 & 278.4973 & 278.498 & 278.458 & 278.4975 & 278.4973 & 279.9344 & 293.7 & 279.0 & 277.5635 \\
\hline $\mathrm{P}(\mathrm{G} 6)$ & 3 & 239.5304 & 239.6315 & 239.632 & 239.670 & 239.6316 & 239.6315 & 239.6610 & 242.3 & 239.7 & 239.6972 \\
\hline $\mathrm{P}(\mathrm{G} 7)$ & 1 & 288.5161 & 288.5845 & 288.585 & 288.608 & 288.5847 & 288.5845 & 287.7275 & 302.8 & 289.0 & 288.9891 \\
\hline $\mathrm{P}(\mathrm{G} 8)$ & 3 & 239.6159 & 239.6315 & 239.632 & 239.634 & 239.6316 & 239.6315 & 239.5520 & 242.3 & 239.7 & 239.4176 \\
\hline $\mathrm{P}(\mathrm{G} 9)$ & 3 & 428.6269 & 428.5216 & 428.518 & 428.479 & 428.5203 & 428.5216 & 427.1485 & 355.1 & 429.2 & 427.3409 \\
\hline $\mathrm{P}(\mathrm{G} 10)$ & 1 & 274.8742 & 274.8667 & 274.866 & 274.880 & 274.8671 & 274.9967 & 275.8687 & 288.8 & 275.2 & 275.6446 \\
\hline$T P(\mathrm{MW} / \mathrm{h})$ & & 2699.9998 & 2699.9999 & 2699.999 & 2700.0001 & 2700.0003 & 2700.1299 & 2700.0002 & 2700.0 & 2702.7 & 2700.0000 \\
\hline$T C(\$ / \mathrm{h})$ & & 623.8090 & 623.8092 & 623.809 & 623.8089 & 623.8090 & 623.8092 & 623.5960 & 626.24 & 625.18 & 623.8140 \\
\hline$S C V$ & & 0.0002 & 0.0001 & 0.0010 & 0.0001 & 0.0003 & 0.1299 & 0.0002 & 0.0000 & 2.7000 & 0.0000 \\
\hline
\end{tabular}

\section{SYSTEM SimULATIONS}

In this chapter, the proposed method has been tested on a realistic 10-unit power system for solving the PEDP of units with MFOs. The cost coefficients together with the smallest and biggest generating capacity limits for every fuel option and load demand 2700MW of this test system are the same as [4]. The MU algorithm was used in QPSO to manage system constraints of the inequality and equality. The computational program was executed on a desktop computer (Intel(R) Core(TM) i7-3770 CPU @ 3.4 GHz with 8G Ram) using FORTRAN-90 program. Setting parameters employed in this test illustrate as the following; the population dimension $m$ is fixed as 20 for the proposed QPSO-MU. The iteration numbers of outer loop and inner loop are set to (outer, inner) as $(10,500)$. Contraction-expansion (CE) coefficientain (8) is set to 1.5 .

The implementation of the proposed algorithm for this test system can be described as follows:

$$
\begin{gathered}
L_{a}(x, v, v)=f(x)+\left\{\left[h_{1}(x)+v_{1}\right]^{2}-v_{1}^{2}\right\} \\
+\sum_{k=1}^{20} \beta_{k}\left\{\left\langle g_{k}(x)+v_{k}\right\rangle_{+}^{2}-v_{k}^{2}\right\} \\
\min _{x=\left(P_{1}, P_{2}, \cdots, P_{10}\right)} f(x)=\sum_{j=1}^{10} F_{j}\left(P_{j}\right)
\end{gathered}
$$

where

$$
F_{j}\left(P_{j}\right)=\left\{\begin{array}{llll}
a_{j 1}+b_{j 1} P_{j}+c_{j 1} P_{j}^{2}, & \text { fuel } 1, & \underline{P}_{j} \leq P_{j} \leq P_{j 1} \\
a_{j 2}+b_{j 2} P_{j}+c_{j 2} P_{j}^{2}, & \text { fuel } 2, & P_{j 1} \leq P_{j} \leq P_{j 2} \\
\vdots & & \vdots \\
a_{j k}+b_{j k} P_{j}+c_{j k} P_{j}^{2}, & \text { fuel } k, & P_{j k-1} \leq P_{j} \leq \bar{P}_{j}
\end{array}\right.
$$

Subject to;

$$
\begin{array}{ll}
h_{1}: & \sum_{i=1}^{10} P_{i}-P_{d}=0 \\
g_{1}: & P_{1}-P_{1}^{\max } \leq \mathrm{O} \\
\vdots & \\
g_{10}: & P_{10}-P_{10}^{\max } \leq 0 \\
g_{11}: & P_{1}^{\min }-P_{1} \leq 0 \\
\vdots & \\
g_{20}: & P_{10}^{\min }-P_{10} \leq 0
\end{array}
$$

The optimization of PEDP of units having MFOs is formed by the objective function (1), the power balance constraint of (3) and the capacity limit constraints of (4). Thus, the optimal cost problem composes of one objective function with ten variable parameters and one equality equation for the power balance constraint. This minimum cost problem consists of one objective function with ten variable parameters, $\left(P_{1} \sim P_{10}\right)$, one equality constraint, $\left(h_{1}\right)$, and twenty inequality constraints, $\left(g_{1} \sim g_{20}\right)$, for the PEDP of units with MFOs. The sum of constraint violations ( $S C V)$ is defined in (16) to inspect effect of the constraint feasibility at the final solution.

$$
S C V=\left|h_{1}\right|+\sum_{k=1}^{20} \max \left\{g_{k}, \quad 0.0\right\}(M W)
$$

For comparison, Table I lists ten optimal results of this practical test system. Results obtained from a synergic predator-prey optimization (SPPO) [18], an integrated of modified shuffled frog leaping algorithm (MSFLA) and global-best harmony search algorithm (GHS), MSFLAGHS [19], QP-ALHN [20], improved particle swarm optimization (IPSO) [21], enhanced augmented Lagrange Hopfield network (EALHN) [22], composite cost function (CCF) [23], chaotic improved honey bee mating optimization (CIHBMO) [24], adaptive Hopfield neural network (AHNN) [7], numerical approach (HM) [4] and the proposed QPSO-MU, are shown in this Table clearly. Although results obtained from SPPO [18], SFLA-GHS [19], QP-ALHN [20], IPSO [21], EALHN [22], CCF [23] and CIHBMO [24] have the less cost than the proposed QPSO-MU. However, such solutions are infeasible ones, because of insufficient or excess demand. The $S C V$ indicates 
that these outputs are infeasible solutions. As seen from the best solution of QPSO-MU with an exact total power (TP) listed in Table I, the $T P$ is $2700.0000 \mathrm{MW}$.

By investigating results presented in Table I, it is observed that the best total cost (TC) utilizing QPSO-MU is 623.8140 $\$ / \mathrm{h}$, which is much less than the best results previously reported in AHNN [7] and HM [4]. Therefore, the result obtained from the proposed QPSO-MU is an optimal and feasible solution. In this Table, all methods have the same fuel type $(F T)$ used for the dispatching generators of this test system.

Table II shows comparisons of the $T C$ and average CPU time $\left(C P U_{-} A V\right)$ obtained from former approaches and the proposed QPSO-MU for this realistic test system. According to the $C P U_{-} A V$ of different methods listed in the Table II, the proposed QPSO-MU is faster than most of the compared algorithms, but slightly slower than EALHN [22]. Consequently, the QPSO-MU is both providing the precise entire power and spending the less $C P U_{-} A V$ than most methods for the PEDP of units with MFOs.

TABLE II: COMPARISONS OF THE TC AND CPU_AV OBTAINED FROM DIFFERENT APPROACHES AND THE PROPOSED QPSO-MU.

\begin{tabular}{cccc}
\hline \hline Method & $T P(\mathrm{MW} / \mathrm{h})$ & $T C(\$ / \mathrm{h})$ & $C P U \_A V(\mathrm{sec})$ \\
\hline HM [4] & 2702.7 & 625.18 & - \\
HNN [5] & 2699.7 & 626.12 & $\sim 60$ \\
ELANN [6] & 2700 & 623.88 & 21.36 \\
AHNN [7] & 2700 & 626.24 & $\sim 4$ \\
ARCGA [9] & 2700 & 623.828 & 0.85 \\
IEP [12] & 2700 & 623.851 & - \\
DE [13] & 2700 & 623.809 & 0.083 \\
MPSO [14] & 2700 & 623.809 & - \\
HRCGA [17] & 2700 & 623.809 & 6.47 \\
HICDEP [18] & 2700 & 623.809 & 0.513 \\
QP-ALHN [20] & 2699.999 & 623.809 & 0.047 \\
IPSO [21] & 2700.0001 & 623.8089 & 0.922 \\
EALHN [22] & 2700.0003 & 623.8090 & 0.013 \\
CIHMBO [24] & 2700.0002 & 623.5960 & 2 \\
QPSO-MU & $\mathbf{2 7 0 0 . 0 0 0 0}$ & $\mathbf{6 2 3 . 8 1 4 0}$ & $\mathbf{0 . 0 4 2}$ \\
\hline \hline
\end{tabular}

\section{CONCLUSION}

The QPSO-MU for solving the PEDP of units with MFOs has been presented herein. The QPSO helps the proposed method efficiently search and refined exploit. The MU assists the presented algorithm both of preventing out of shape of the ALF and leading to difficulty of solution finding. The presented approach combines the QPSO with the MU that possess benfits of taking a large area of punishment parameters and a small population. A practical $10-\mathrm{G}$ system is used to compare the proposed QPSO-MU with previous methods. Simulation results show that the proposed algorithm is superior to previous approaches in solution quality for solving the PEDP of units with MFOs. Contributions of this paper are the QPSO precisely finds the optimal answers in the economic dispatch procedure, and the MU validly handles in system constraints management of power system.

\section{ACKNOWLEDGMENT}

I acknowledge and express thanks for the monetary assistance given to this paper by the Ministry of Science Technology, Taiwan, R.O.C. under Grant No. MOST 1063011-F-252-001.

\section{REFERENCES}

[1] A. J. Wood and B. F. Wollenberg, Power Generation Operation and Control, 2nd ed, Wiley, New York, 1996.

[2] A. Chakrabarti and S. Halder, Power System Analysis Operation and Control, 3rd Edition, PHI, New Delhi, 2010.

[3] R. R. Shoults and M.M. Mead, "Optimal estimation of piece-wise linear incremental cost curves for EDC," IEEE Trans. Power Appar. Syst. PAS-103, vol. 6, pp. 1432-1438, 1984.

[4] C. E. Lin and G. L. Viviani, "Hierarchical economic dispatch for piecewise quadratic cost functions," IEEE Trans. Power App. Syst., PAS-103, no. 6, pp. 1170-1175, 1984.

[5] J. H. Park, Y. S. Kim, I. K. Eom, and K. Y. Lee, "Economic load dispatch for piecewise quadratic cost function using Hopfield neural network," IEEE Trans. Power Syst., vol. 8, no. 3, pp. 1030-1038, 1993.

[6] S. C. Lee and Y. H. Kim, "An enhanced Lagrangian neural network for the ELD problems with piecewise quadratic cost functions and nonlinear constraints," Electr. Power Syst. Res., vol. 60, no. 3, pp. 167-177, 2002.

[7] K. Y. Lee, A. Sode-Yome, and J. H. Park, "Adaptive Hopfield neural networks for economic load dispatch," IEEE Trans. Power Syst., vol. 13, no. 2, pp. 519-526, May 1998.

[8] K. Y. Lee, F. M. Nuroglu, and A. Sode-Yome, "Real power optimization with load flow using adaptive Hopfield neural network," Eng. Intell. Syst., vol. 8, no. 1, pp. 53-58, 2000.

[9] N. Amjady and H. Nasiri-Rad, "Solution of nonconvex and nonsmooth economic dispatch by a new adaptive real coded genetic algorithm," Expert Syst. Appl., vol. 37, no. 7, pp. 5239-5245, 2010.

[10] C. L. Chiang and C. T. Su, "Adaptive-improved genetic algorithm for the economic dispatch of units with multiple fuel options," Cybern. Syst.: An Internat. J., vol. 36, no. 7, pp. 687-704, 2005.

[11] T. Jayabarathi, K. Jayaprakash, D. N. Jeyakumar, and T. Raghunathan, "Evolutionary programming techniques for different kinds of economic dispatch problems," Electr. Power Syst. Res., vol.73, no. 2, pp. 169-176, 2005.

[12] Y. M. Park, J. R. Wong, and J. B. Park, "A new approach to economic load dispatch based on improved evolutionary programming," Eng. Intell. Syst. Elect. Eng. Commun., vol. 6, no. 2, pp. 103-110, 1998.

[13] N. Noman and H. Iba, "Differential evolution for economic load dispatch problems," Electr. Power Syst. Res., vol. 78, no. 8, pp. 13221331,2008

[14] J. B. Park, K. S. Lee, and K. W. Lee, "A particle swarm optimization for economic dispatch with nonsmooth cost function," IEEE Trans. Power Syst., vol. 12, no. 1, pp.34-42, 2005.

[15] W. M. Lin, F. S. Cheng, and M. T. Tsay, "Nonconvex economic dispatch by integrated artificial intelligence," IEEE Trans. Power Syst., vol. 16, no. 2, pp. 307-311,2001.

[16] S. Baskar, P. Subbaraj, and M. V. C. Rao, "Hybrid real coded genetic algorithm solution to economic dispatch problem," Comput. Electr. Eng., vol. 29, no. 3, pp. 407-419, 2003.

[17] R. Balamurugan and S. Subramanian, "Hybrid integer coded differential evolution-dynamic programming approach for economic load dispatch with multiple fuel options," Energy Convers. Manag., vol. 49, no. 4, pp. 608-614, 2008.

[18] N. J. Singh, J. S. Dhillon, D. P. Kothari, "Synergic predator-prey optimization for economic thermal power dispatch problem," Applied Soft Computing, vol. 43, pp. 298-311, 2016.

[19] K. Vaisakha and A. Srinivasa Reddy, "MSFLA/ GHS/ SFLA-GHS/ SDE algorithms for economic dispatch problem considering multiple fuels and valve point loadings," Applied Soft Computing, vol. 13, pp. 4281-4291, 2013.

[20] Vo Ngoc Dieu and Peter Schegner, "Augmented Lagrange Hopfield network initialized by quadratic programming for economic dispatch with piecewise quadratic cost functions and prohibited zones," Applied Soft Computing, vol. 13, pp. 292-301, 2013.

[21] A. K. Barisal, "Dynamic search space squeezing strategy based intelligent algorithm solutions to economic dispatch with multiple fuels," Electrical Power and Energy Systems, vol.45, pp. 50-59, 2013.

[22] Vo Ngoc Dieu and W. Ongsakul, "Economic dispatch with multiple fuel types by enhanced augmented Lagrange Hopfield network," Applied Energy, vol. 91, pp. 281-289, 2012.

[23] R. Anandhakumar and S. Subramanian, "Economic Dispatch with Multiple Fuel Options Using CCF," Energy and Power Engineering, vol. 3, pp. 113-119, 2011.

[24] T. Niknam, H. D. Mojarrad, H. Z. Meymand, and B. B. Firouzi, "A new honey bee mating optimization algorithm for non-smooth economic dispatch," Energy, vol. 36, pp. 896-908, 2011. 
[25] R. C. Eberhart and Y. Shi, "A modified swarm optimizer," In: Proceedings of the 1998 IEEE international conference of evolutionary computation, pp. 1945-1950, 1998.

[26] J. Kennedy, and R. Eberhart, "A new optimizer using particle swarm theory," Proceedings of the Sixth International Symposium on Micro Machine and Human Science, pp. 39-43, 1995.

[27] Y. Wang, Y. Yang, "Particle swarm with equilibrium strategy of selection for multi-objective optimization," European Journal of Operational Research, vol. 200, issue 1, pp. 187-197, Jan. 2010.

[28] J. Chang, "A robust adaptive array beamformer using particle swarm optimization for space-time code division multiple access systems," Inf. Sci., vol. 278, pp. 174-186, 2014.

[29] A. Ouyang, K. Li, T. K. Truong, A. Sallam, and E. H. M. Sha, "Hybrid particle swarm optimization for parameter estimation of Muskingum model," Neural Comput. \& Applic., vol. 25, issue. 7-8, pp. 17851799, 2014.

[30] A. Ouyang, Z. Tang, X. Zhou, Y. Xu, G. Pan, and K. Li, "Parallel hybrid PSO with CUDA for LD heat conduction equation," Comput. Fluids, vol. 110, pp. 198-210, 2015.

[31] J. Zhao, M. Lin, D. Xu, L. Hao, and W. Zhang, "Vector control of a hybrid axial field flux-switching permanent magnet machine based on particle swarm optimization," IEEE Trans. Magn., vol. 51, issue11, Nov. 2015.

[32] P. Regulski, D. Vilchis-Rodriguez, S. Djurovic, and V. Terzija, "Estimation of composite load model parameters using an improved particle swarm optimization method," IEEE Trans. Power Deliv., vol. 30, no. 2, pp. 553-560, 2015.

[33] B. Mohammadi-Ivatloo, M. Moradi-Dalvand and A. Rabiee, "Combined heat and power economic dispatch problem solution using particle swarm optimization with time varying acceleration coefficients," Electric Power Syst. Res., vol. 95, pp. 9-18, 2013.

[34] F. Bergh, "An Analysis of Particle Swarm Optimizers," Ph.D. thesis, University of Pretoria, 2001.

[35] F. Bergh, A. Engelbrecht, "A new locally convergent particle swarm optimizer," IEEE Int. Conf. Syst. Man Cybern. Vol. 3, 2002.

[36] J. Sun, B. Feng, W. Xu, "Particle swarm optimization with particles having quantum behavior," in: Proceedings of the 2004 Congress on Evolutionary Computation, CEC2004, pp. 326-331, 2004.

[37] J. Sun, X. Xu, V. Palade, W. Fang, C. Lai, and W. Xu, "Convergence analysis and improvements of quantum-behaved particle swarm optimization," Inf. Sci., vol. 193, no. 15, pp. 81-103, 2012.

[38] J. Sun, W. B. Xu, and B. Feng, "Adaptive parameter control for quantum-behaved particle swarm optimization on individual level," In: Proceedings of the 2005 IEEE international conference on systems, man and cybernetics, vol. 4, pp. 3049-3054, 2005.

[39] F. S. Levin, "An introduction to quantum theory," Cambridge: Cambridge University Press, 2002.
[40] T. Liu, L. C. Jiao, W. P. Ma, and R. H. Shang, "Quantum-behaved particle swarm optimization with collaborative attractors for nonlinear numerical problems," Commun. Nonlinear Sci. Numer. Simulat., vol. 44, pp. 167-183, 2017.

[41] Y. Y Li, X. Bai, L. C Jiao, and Y. Xue, "Partitioned-cooperative quantum-behaved particle swarm optimization based on multileve thresholding applied to medical image segmentation," Applied Soft Computing, vol. 56, pp. 345-356, 2017.

[42] Z. k. Feng, W. J Niu, and C. T Cheng, "Multi-objective quantumbehaved particle swarm optimization for economic environmental hydrothermal energy system scheduling," Energy, vol. 131, pp. 165178, 2017.

[43] T. Liu, L. C Jiao, W. P Ma, J. J Ma, and R. H Shang, "Cultural quantum-behaved particle swarm optimization for environmental/economic dispatch," Applied Soft Computing, vol. 48, pp. 597-611, 2016

[44] W. Fang, J. Sun, H. H Chen, and X. J. Wu, "A decentralized quantuminspired particle swarm optimization algorithm with cellular structured population," Information Sciences, vol. 330, pp. 19-48, 2016.

[45] M. Clerc and J. Kennedy, "The particle swarm: explosion, stability and convergence in a multi-dimensional complex space," IEEE Trans Evol. Comput., vol. 6, pp.58-73, 2002.

[46] Z. Michalewicz and M. Schoenauer, "Evolutionary algorithms for constrained parameter optimization problems," Evolutionary Computation, vol. 4, no. 1, pp.1-32, 1996.

[47] M. J. D. Powell, "Algorithms for nonlinear constraints that use Lagrangian function,” Math. Programming, vol. 14, pp 224-248, 1978.

[48] C. L. Chiang, C. T. Su, and F. S. Wang, "Augmented Lagrangian method for evolutionary optimization of mixed-integer nonlinea constrained problems," International Mathematics Journal, vol.2, no. 2, pp. 119-154, 2002

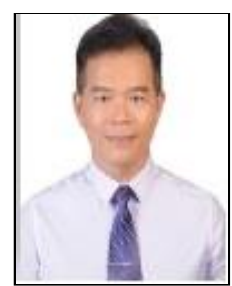

C. L. Chiang received his M.S. degree from Automatic Control Engineering, Feng Chia University, Taichung, Taiwan in 1991, and Ph. D. degree from Institute of Electrical Engineering, National Chung Cheng University, Chia-Yi, Taiwan in 2004.

$\mathrm{He}$ is now a professor of Nan Kai University of Technology, Nan-Tou, Taiwan, ROC. His research interests are in the control theory, applications of the optimization, evolutionary algorithms and power economic dispatch. 